\title{
Molecular characterization and virulence of an alphaherpesvirus isolated from a BoHV1 gB-seropositive and gE-seronegative Italian buffalo
}

\author{
Preziuso S. ${ }^{\text {, }}$ Marenzoni M.L. ${ }^{\mathrm{b}}$, Thiry J. ${ }^{\mathrm{c}}$, Thiry E. ${ }^{\mathrm{c}}$, Cuteri V. ${ }^{\mathrm{a}, *}$ \\ a School of Biosciences and Veterinary Medicine, University of Camerino, Matelica, Italy \\ ${ }^{\mathbf{b}}$ Department of Veterinary Medicine, University of Perugia, Perugia, Italy \\ ${ }^{\mathrm{c}}$ Veterinary Virology and Animal Viral Diseases, Fundamental and Applied Research for Animal \& Health Centre, Faculty of Veterinary Medicine, University of Liège, Liège, \\ Belgium
}

\section{A R T I C L E I N F O}

\section{Keywords:}

Herpesvirus

Buffalo

Experimental infection

Serology

PCR

Phylogenesis

\begin{abstract}
A B S T R A C T
During a serological survey, 157 out of 681 unvaccinated buffaloes resulted seropositive for bovine alphaherpesvirus 1 (BoHV1) glycoprotein B (gB) and seronegative for BoHV1 glycoprotein E (gE). These serological results were generally expected in animals vaccinated with a BoHV1 gE-deleted vaccine but not in unvaccinated animals. Seroneutralization tests on 36 selected sera detected neutralizing antibody titers more than three times higher for BuHV1 than for BoHV1. In order to investigate the virus, one of these buffaloes was injected with dexamethasone, and from nasal and vaginal swabs collected at different time points, a ruminant herpesvirus was isolated, characterized and also detected by PCR. Restriction enzyme analysis, sequencing and phylogenic analysis of gB and gD genes showed that the virus was genetically similar but not identical to BuHV1 strain b6. Intranasal inoculation of the virus in a healthy seronegative buffalo resulted in a mild and transient upper respiratory disease; the virus was isolated from clinical specimens and DNA was detected by PCR in nasal and vaginal swabs up to 9 days after infection. Further investigations should be aimed at sequencing the whole viral genome and at evaluating the host-range of this virus. Specific tests are needed to discriminate infections by different ruminant herpesviruses and to improve eradication programs of infectious bovine rhinotracheitis/infectious pustular vulvovaginitis in cattle.
\end{abstract}

\section{Introduction}

Herpesviruses are DNA viruses characterized by a capacity to induce latent infection. Among alphaherpesviruses infecting ruminants, the prototype is Bovine alphaherpesvirus 1 (BoHV1), a pathogen of cattle associated mainly with infectious bovine rhinotracheitis (IBR) and infectious pustular vulvovaginitis (IPV). In addition, several alphaherpesviruses related to BoHV1 have been isolated and characterized in domestic and wild ruminants, showing usually low virulence (Thiry et al., 2006 and 2007). In Italy, water buffalo breeding is consistent, especially in South and Central regions because of typical production, like buffalo Mozzarella cheese. Mixed breeding of cattle and buffalo also exists. However, a Bubaline alphaherpesvirus 1 (BuHV1) has been found in a water buffalo fetus associated with abortion in Italy (Amoroso et al., 2013). Further investigations in Italy showed a high seroprevalence for BuHV1 in Piedmont (Northern Italy) and Campania (Southern Italy) regions, where IBR control programs have been implemented (Caruso et al., 2016). In order to enable control and eradication of IBR diseases in the European Union, rules for the intra-
Community movement of bovine animals have been implemented. Although control measures should be directed to the eradication of the infection rather than to the suppression of disease, the use of gE-deleted BoHV1 vaccines is allowed in bovine animals (Decision 2004/558/CE). The use of DIVA (Differentiating Infected from Vaccinated Animals) vaccines is favored because they do not interfere with eradication programs based on detection of infected animals. In this case, gB- and gE-seropositive animals are considered infected with BoHV1, while gBpositive and gE-negative animals are considered vaccinated with a $\mathrm{gE}$ deleted vaccine. However, eradication programs could fail if animals are infected by a gE-positive BuHV1 causing interference in the interpretation of test results.

This paper describes the case occurred in a buffalo farm never vaccinated with a gE-deleted BoHV1 vaccine but resulted seropositive for anti-BoHV1 gB antibodies and seronegative for anti-gE antibodies. The aims of this study were i) to investigate the cause of these serological results by isolation and characterization of the virus from a gBpositive/gE-negative buffalo and ii) to evaluate the virulence and the kinetic of viral excretion in a seronegative experimentally infected

\footnotetext{
* Corresponding author at: Circonvallazione 93-95, 62024, Matelica, MC, Italy.

E-mail address: vincenzo.cuteri@unicam.it (V. Cuteri).
} 
buffalo.

\section{Materials and methods}

\subsection{Serological screening}

Sera samples were collected for an epidemiological survey from 681 buffaloes reared in 8 different herds in Central Italy. The animals were not vaccinated for BoHV1 and gE deleted BoHV1 vaccine had been never used in these farms. Sera were tested by two commercial blocking ELISA tests to detect antibodies anti-BoHV1-gB and anti-BoHV1-gE (IBR gB/gE IDEXX Herd Check Laboratories). BoHV1 infected animals are supposed to be positive to both tests, uninfected animals are supposed to be negative to both tests, and animals vaccinated with a BoHV1-gEdeleted vaccine are supposed to be seropositive for $\mathrm{gB}$ but seronegative for $\mathrm{gE}$. The serological tests were carried out following the manufacturer's instructions.

On the basis of the ELISA results, selected sera were subsequently tested by seroneutralization to detect anti-BuHV1 and anti-BoHV1 antibodies.

\subsection{Seroneutralization test}

Seroneutralization test was performed as follows. Each sample was diluted 1:2 in minimal essential medium (MEM) containing 10\% fetal bovine serum and two-fold serial dilutions of sera in MEM were incubated in sterile 96-well microtitre plates for $1 \mathrm{~h}$ at $37^{\circ} \mathrm{C}$ with an equal volume of the diluted virus $\left(100-200\right.$ TCID $_{50}$ of BoHV1 LA strain (Castrucci et al., 1984)). All sera were incubated separately with BuHV1 and with BoHV1. Approximately $1.5 \times 10^{4}$ MDBK cells were added to each well and the plates were incubated for 3-5 days at $37^{\circ} \mathrm{C}$ in a humid atmosphere of $5 \% \mathrm{CO}_{2}$ in air. Plates were checked and read daily. Final reading was performed the 5 th day. The results were expressed as the reciprocal of the dilution of the serum that neutralized the virus in $50 \%$ of the wells. Positive and negative control sera and controls for the cell viability, BuHV1 and BoHV1 infectivity, and test serum cytotoxicity were included in each assay. The control immune sera used for the seroneutralization test were obtained from one buffalo and from one cattle inoculated respectively with BuHV1 and with BoHV1.

\subsection{Virus reactivation and isolation}

In order to reactivate the herpesvirus from latency, the buffalo ID 810 was selected, on the basis of seroneutralization results, for pharmacological treatment similarly as previously reported (De Carlo et al., 2004). In particular, sodium dexamethasone-21-phosphate (Desashock, Fort Dodge Animal Health) at a dosage of $4 \mathrm{mg} / \mathrm{kg}$ body weight was injected intramuscularly daily for five days (from day 1 to day 5). Nasal and vaginal swabs were collected daily for 11 days (from day 5 to day 15) for viral isolation and PCR. Immediately after sampling, the swabs collected for viral isolation were put in a tube with MEM, while swabs collected for PCR analysis were transported in tubes without MEM. Once arrived in the laboratory, samples in MEM were filtered $(0.45 \mu \mathrm{m})$ and $100 \mu \mathrm{l}$ inoculated into each well of 24 -well plates containing confluent MDBK cell cultures. The plates were incubated at $37^{\circ} \mathrm{C}$ in modified atmosphere with $5 \% \mathrm{CO}_{2}$ and daily checked for cytopathic effects. Seven days after inoculation, the monolayer was frozen and thawed, and the supernatant was inoculated in another plate containing a MDBK monolayer up to a total of three passages. MDBK cultures showing cytopathic effects were tested by PCR. The virus isolate was named IT08M134.

\subsection{DNA extraction and $P C R$}

DNA was extracted from $100 \mu \mathrm{l}$ cell cultures and from all nasal and vaginal swabs by using the commercial kit Genomic DNA isolation kit (Norgen Biotek Corp., Thorold, ON, Canada) following the manufacturer's instructions and eluting the DNA in $100 \mu$ f final volume. DNA was also obtained from cell cultures infected with BoHV1 strain Cooper, which has been used as positive control.

PCR with primers CR30 and CR31 were used to amplify and sequence a $444 \mathrm{bp}$ region of the gB gene (UL27) of all ruminant alphaherpesviruses related to BoHV1 (Ros and Belák, 1999). Further PCRs were carried out to detect and sequence genes codifying for the $\mathrm{gD}$ (primers CR52/CR54) (Ros and Belák, 1999; De Carlo et al., 2004), gB (gB-1/gB-2 and $\mathrm{gBN}-1 / \mathrm{gBN}-2), \mathrm{gC}(\mathrm{gC}-1 / \mathrm{gC}-2)$ and $\mathrm{gE}(\mathrm{gE}-1 / \mathrm{gE}-2$ and gEN-1/gEN-2) of BoHV1 (Fuchs et al., 1999).

PCRs were carried out in $100 \mu \mathrm{l}$ final volume containing $50 \mu \mathrm{l}$ of Taq PCR mastermix (Qiagen, Germany), 50 pmol of the forward and of the reverse primers, and $2 \mu \mathrm{l}$ of DNA. The mixture for the nested PCR was the same but products of the first PCRs were used as a template. PCR grade water instead of DNA was used as a negative control. PCRs with gE-1/gE-2 primers and gEN-1/gEN-2 primers were carried out with and without addition of $5 \%(\mathrm{vol} / \mathrm{vol})$ dimethyl sulfoxide. The amplification programs consisted of $95^{\circ} \mathrm{C}$ for $5 \mathrm{~m}, 35$ cycles of $95^{\circ} \mathrm{C}$ for $1 \mathrm{~m}, 52^{\circ} \mathrm{C}$ (primers CR52/CR54) or $60^{\circ} \mathrm{C}$ (primers CR30/CR31) for $1 \mathrm{~m}, 72{ }^{\circ} \mathrm{C}$ for $1 \mathrm{~m}$, and final extension at $72{ }^{\circ} \mathrm{C}$ for $7 \mathrm{~m}$. PCRs for the $\mathrm{gB}, \mathrm{gC}$ and $\mathrm{gE}$ genes were carried out as previously described (Fuchs et al., 1999), but separate single reactions instead of a multiplex PCR reaction in order to sequence the products obtained were performed.

Both first and second reaction products were analyzed by electrophoresis of $10 \mu \mathrm{l}$ of reaction products in $1.5 \%$ agarose gels containing $0.5 \mathrm{mg} / \mathrm{ml}$ of ethidium bromide with Tris-borate-EDTA buffer $(89 \mathrm{mM}$ Tris, $89 \mathrm{mM}$ boric acid, $2 \mathrm{mM}$ EDTA pH: 8.3). Images were visualized on a UV transluminator and captured by the Kodak Gel Logic 100 system.

\subsection{Restriction enzyme analysis (REA)}

Viral DNA obtained by a cell culture with cytopathic effects and positive by PCR was submitted to BamHI and BstEII restriction endonucleases (New England Biolabs, England, United Kingdom) (Thiry et al., 2007). The digestion products were electrophoresed in a $0.7 \%$ Tris Acetate EDTA agarose gel for $18 \mathrm{~h}$ at $50 \mathrm{~V} / \mathrm{cm}$ and $500 \mathrm{~mA}$. SmartLadder (10 kb; Eurogentec, Liège, Belgium) as molecular mass marker was used. DNA of other ruminant alphaherpesviruses was used as control (Thiry et al., 2007).

\subsection{Sequencing of the PCR products and phylogenetic analysis}

PCR products obtained by primers $\mathrm{gB}-1 / \mathrm{gB}-2$, CR30/CR31 and CR52/CR54 were treated with ExoSAP-IT and were sequenced on both strands by an external laboratory (BMR Genomics, Padua, Italy). Primers gB-1/gB-2 and CR30/CR31 bind the UL27 sequence of BuHV1 strain b6 (KU936049.1) at positions 56932-56951/57390-57409 and 56707-56726/57130-57150 respectively and amplify two segments which partially overlap each other. The sequences obtained were visually checked and were analyzed by NIH BLAST. All sequences of the UL27 gene (encoding the glycoprotein B) and of the UL6 gene (encoding for the $\mathrm{gD}$ ) of ruminant herpesviruses available in GenBank and EMBL were downloaded and aligned with the sequences obtained in our study. Sequences were aligned using Clustal X (Larkin et al., 2007) and manual editing was performed with BioEdit version 7.2.5 (Hall, 1999). MEGA version 7.0 was used to select the simplest evolutionary model that adequately fitted the sequence data (Kumar et al., 2016). The model with the lowest Bayesian Information Criterion (BIC) was considered to describe the substitution pattern the best. On the basis of the BIC values obtained, the model Tamura- 3 parameter with a discrete Gamma distribution $(+\mathrm{G})$ with 5 parameters was used (Tamura, 1992). The neighbor-joining algorithm (NJ) and the maximum likelihood (ML) method were estimated for phylogeny. In both analyses, 1000 bootstrap replicates were used. 


\subsection{Virus inoculation in a seronegative buffalo}

A female buffalo resulted seronegative for BoHV1 and for BuHV1 at three tests performed weekly was selected for experimental infection with the virus IT08M134. The buffalo was kept separately from other animals and was clinically monitored for seven days. At T0 serum, nasal and vaginal swabs were collected from the buffalo, which was subsequently infected by the nasal route with $1 \mathrm{ml} /$ nostril of viral suspension

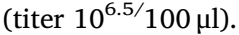

The animal was clinically examined and the body temperature was recorded daily for 21 days. Nasal and vaginal swabs were collected daily for 21 days for viral isolation and for PCR. On the basis of preliminary comparative evaluations, nested PCR with primers gB-1/gB-2 and $\mathrm{gBN}-1 / \mathrm{gBN}-2$ for detecting the $\mathrm{gB}$ gene showed higher sensitivity than the other PCRs and was used for testing these samples (data not showed). However, the PCR protocol was optimized in order to maximize its sensitivity. In particular, the new protocol consisted of $95^{\circ} \mathrm{C}$ for $5 \mathrm{~m}, 35$ cycles of $95^{\circ} \mathrm{C}$ for $1 \mathrm{~m}, 69^{\circ} \mathrm{C}$ (primers gB-1/gB-2) or $55^{\circ} \mathrm{C}$ (primers gBN-1/gBN-2) for $1 \mathrm{~m}, 72^{\circ} \mathrm{C}$ for $1 \mathrm{~m}$, and final extension at $72{ }^{\circ} \mathrm{C}$ for $7 \mathrm{~m}$. Blood sera were collected weakly for seroneutralization tests.

\section{Results}

\subsection{Serological screening and seroneutralization tests}

A total of 555 out of 681 sera (81.5\%) resulted positive for anti-gB antibodies, $102(15.0 \%)$ were negative and $24(3.5 \%)$ were doubtful. Out of the 555 anti-gB positive sera, $157(28.3 \%)$ were negative for anti-gE antibodies, 355 (64.0\%) were gE-positive and 43 (7.7\%) were doubtful. All 8 farms had at least one animal with anti-gB antibodies.

Randomly selected sera resulted gB-positive and gE-positive $(\mathrm{n}=10)$, gB-positive and gE-doubtful $(\mathrm{n}=10)$, gB-positive and gEnegative $(n=10)$, gB-negative and gE-positive $(n=3)$, gB-negative and gE-doubtful $(n=3)$ were tested by seroneutralization tests.

All sera gB-positive and gE-positive, all sera gB-positive and gEdoubtful and all but two sera gB-positive and gE-negative showed neutralizing antibody titers more than three times higher for BuHV1 than for BoHV1 (Table 1). All gB-negative and gE-doubtful sera and 2 out of 3 gB-negative and gE-positive sera did show neutralizing antibodies against neither BoHV1 nor BuHV1, while one serum showed titers more than three times higher for BuHV1 than for BoHV1.

\subsection{Virus reactivation, isolation and PCR}

After treatment with sodium dexamethasone-21-phosphate, the infected buffalo showed fever, clear ocular and nasal discharge, mild cough and dyspnea.

Cytopathic effects suggestive of herpesvirus infection were observed in cell cultures inoculated with nasal and vaginal swabs. Results of viral isolation and nested PCR obtained from infected cell culture as well as nasal and vaginal swabs collected from 5 to 15 days after glucocorticoid treatment are reported in Table 2. Nested PCR results were positive with primers $\mathrm{gB}-1 / \mathrm{gB}-2$ and $\mathrm{gBN}-1 / \mathrm{gBN}-2$ but not with primers $\mathrm{gE}-1 /$ gE-2 and gEN-1/gEN-2.

\subsection{Restriction enzyme analysis (REA) on viral DNA}

REA tests demonstrated that the isolate IT08M134 was genetically similar but not identical to BuHV1 b6 and distinct from BoHV1 (1.1 Lam and 1.2a K22) (Fig. 1). Its REA pattern was also distinct from the other related ruminant alphaherpesviruses namely BoHV1, caprine alphaherpesvirus 1, cervid alphaherpesviruses 1 and 2 (Thiry et al., 2007).
Table 1

Cross-serum neutralisation assay of different categories of serum samples against BoHV1 and BuHV1.

\begin{tabular}{|c|c|c|}
\hline \multirow[t]{2}{*}{ Blocking ELISA results } & \multicolumn{2}{|c|}{ Neutralising antibody titre against } \\
\hline & BoHV1 & BuHV1 \\
\hline \multirow[t]{10}{*}{ gB-positive/gE-positive } & $1: 8$ & $1: 64$ \\
\hline & $1: 8$ & $1: 128$ \\
\hline & $1: 16$ & $1: 128$ \\
\hline & $1: 16$ & $1: 128$ \\
\hline & $1: 16$ & $1: 256$ \\
\hline & $1: 16$ & $1: 256$ \\
\hline & $1: 32$ & $1: 1024$ \\
\hline & $1: 32$ & $>1: 2048$ \\
\hline & $1: 256$ & $1: 512$ \\
\hline & $1: 512$ & 1:1024 \\
\hline \multirow[t]{10}{*}{ gB-positive/gE-doubtful } & $1: 16$ & $1: 512$ \\
\hline & $1: 64$ & $1: 512$ \\
\hline & $1: 64$ & $1: 256$ \\
\hline & $1: 32$ & $1: 512$ \\
\hline & $1: 32$ & $1: 512$ \\
\hline & $1: 64$ & $1: 128$ \\
\hline & $1: 64$ & $1: 128$ \\
\hline & $1: 64$ & $>1: 2048$ \\
\hline & $1: 32$ & $1: 128$ \\
\hline & $1: 64$ & $1: 512$ \\
\hline \multirow[t]{10}{*}{ gB-positive/gE-negative } & $1: 8$ & $1: 64$ \\
\hline & $1: 8$ & $1: 512$ \\
\hline & $1: 8$ & $>1: 2048$ \\
\hline & $1: 16$ & $1: 64$ \\
\hline & $1: 64$ & $1: 512$ \\
\hline & $1: 64$ & $>1: 2048$ \\
\hline & $1: 128$ & $1: 512$ \\
\hline & $1: 128$ & $1: 512$ \\
\hline & $1: 128$ & $>1: 2048$ \\
\hline & $1: 128$ & $>1: 2048$ \\
\hline \multirow[t]{3}{*}{ gB-negative/gE-positive } & $<1: 2$ & $<1: 2$ \\
\hline & $<1: 2$ & $<1: 2$ \\
\hline & $1: 128$ & $1: 512$ \\
\hline \multirow[t]{3}{*}{ gB-negative/gE-doubtful } & $<1: 2$ & $<1: 2$ \\
\hline & $<1: 2$ & $<1: 2$ \\
\hline & $<1: 2$ & $<1: 2$ \\
\hline
\end{tabular}

Table 2

Viral isolation and PCR results on nasal and vaginal swab samples collected from the buffalo 810 treated with sodium dexamethasone-21-phosphate. Legend: NS = positivity in nasal swab samples; VS = positive in vaginal swab samples; neg = negativity in both nasal and vaginal swab samples.

\begin{tabular}{lll}
\hline Days* & Viral isolation & PCR \\
\hline 5 & neg & NS, VS \\
6 & VS & NS, VS \\
7 & NS, VS & NS, VS \\
8 & VS & NS, VS \\
9 & VS & NS, VS \\
10 & NS, VS & NS, VS \\
11 & VS & NS, VS \\
12 & VS & NS, VS \\
13 & NS, VS & NS, VS \\
14 & NS, VS & NS, VS \\
15 & neg & NS, VS \\
\hline
\end{tabular}

*Days after the first dexamethasone inoculation.

\subsection{Virus inoculation in a seronegative buffalo}

After virus inoculation, the buffalo showed transient serous nasal discharge for 7 days. Fever was not recorded and rectal temperature was about $38.2-38.5^{\circ} \mathrm{C}$. Virus was isolated from nasal swabs from day 2 to day 4 and from vaginal swabs from day 1 to day 5. PCR was positive on nasal swabs and vaginal swab samples collected from day 1 to day 9 


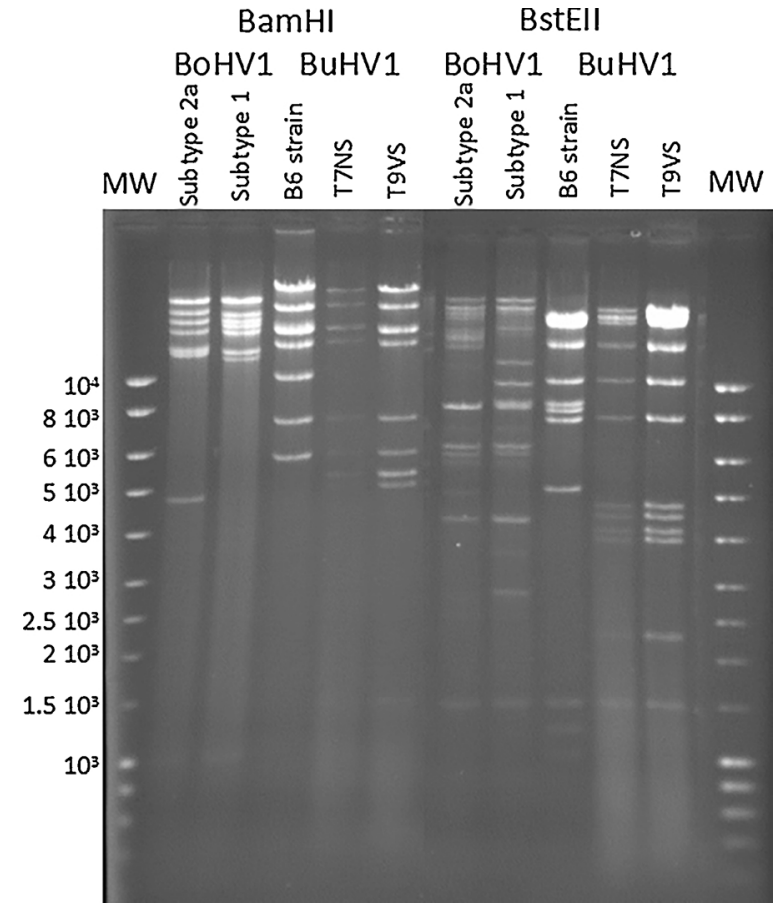

Fig. 1. Restriction endonuclease analysis patterns (enzymes BamHI and BstEII) of bubaline herpesvirus 1 (BuHV1) isolated from experimentally reactivated buffalo IT08M134. T7NS: day 7 nasal swab; T9VS: day 9 vaginal swab. The profiles of the reactivated BuHV1 are clearly different from BoHV1 subtype 1 (Lam strain) and subtype 2 (K22 strain) and the Italian BuHV1 b6. MW: molecular weight marker (base pairs)

Table 3

Viral isolation and PCR results on nasal and vaginal swab samples collected from the buffalo inoculated intranasally with BuHV1 strain IT08M134. Legend: NS = positivity in nasal swab samples; VS = positivity in vaginal swab samples; neg $=$ negativity in both nasal and vaginal swab samples.

\begin{tabular}{lll}
\hline Days* & Viral isolation & PCR \\
\hline 0 & neg & neg \\
1 & VS & NS, VS \\
2 & NS, VS & NS, VS \\
3 & NS, VS & NS, VS \\
4 & NS, VS & NS, VS \\
5 & VS & NS, VS \\
6 & neg & NS, VS \\
7 & neg & NS, VS \\
8 & neg & NS, VS \\
9 & neg & NS, VS \\
10 & neg & neg \\
11 & neg & neg \\
12 & neg & neg \\
13 & neg & neg \\
14 & neg & neg \\
15 & neg & neg \\
\hline
\end{tabular}

*Days after virus inoculation.

(Table 3).

\subsection{Sequencing of the PCR products and phylogenetic analysis}

Single PCRs with primers gB-1/gB-2, CR30/CR31 and CR52/CR54 gave expected products using DNA of infected cell cultures, swabs collected from immunosuppressed buffalo and swabs of buffalo infected with the virus IT08M134. Editing of UL27 overlapping sequences obtained with primers $\mathrm{gB}-1 / \mathrm{gB}-2$ and CR30/CR31 gave sequences about 680 bp long (GenBank accession numbers MH253680 and MH253681) (Fig. S1). The sequences obtained from infected cells and from swab samples were identical each others. BLAST analysis of the $\mathrm{gB}$ and $\mathrm{gD}$ sequences showed higher identity with BuHV1 strain b6 (99\% and $100 \%$ respectively). Phylogenetic analyses assessed the relationship between the strain IT08M134 and other ruminant alphaherpesviruses (Fig. 2a and b). IT08M134 clustered with BuHV1 strain b6, and it was more related to BoHV5 than to BoHV1. By analyzing multiple nucleotide sequence alignments in the UL27 partial sequence, strain IT08M134 differed from BuHV1 strain b6 by 3 out of 683 bases, from BoHV5 strain SV507/99 by 14 bases and from BoHV1 strain Cooper by 28 bases (Fig. S1). Despite these substitutions, a few amino acid changes were found. Indeed, there was only one amino acid substitution between IT08M134 and BuHV1 strain b6, 3 substitutions between IT08M134 and BoHV5 strain SV507/99 and 9 substitutions between IT08M134 and BoHV1 strain Cooper (Fig. S2). Only a partial comparison was possible with an other Italian bubaline isolate (GenBank accession number AJ496607) because only a 414 bp sequence was available. Strain IT08M134 differed from this BuHV1 strain by 2 out of $338 \mathrm{nt}$, corresponding to only one amino acid substitution (Fig. S1 and S2). UL6 sequence of IT08M134 was identical to BuHV1 strain b6, but differed from BoHV5 strain SV507/99 by 10 bases out of 558 and from BoHV1 strain Cooper by 45 bases.

\section{Discussion}

IBR is a OIE-listed bovine disease causing significant economic losses in many countries. In European Union different control measures are adopted depending on the Member State on the basis of the prevalence of BoHV1 infection. Many countries allow and carry out vaccinations, while vaccination is prohibited in regions and countries where the disease has been eradicated. Screening, surveillance, precautions at borders and culling of infected animals are often carried out as eradication policy. In order to reduce the prevalence of IBR, the European Community approved eradication programs to obtain BoHV1free holdings (Decision 2004/558/EC). Among others, specific serological tests are used to discriminate among uninfected, vaccinated with a gE-deleted vaccine or infected animals. In particular, bovine animals for breeding and production coming from Member States and destined for intra-Community movements shall be $\mathrm{gE}$ - seronegative in case of vaccinated animals, or seronegative for the entire BoHV1 in case of unvaccinated animals. In the present study, a buffalo infected with BuHV1 strain IT08M134 resulted gB-positive and gE-negative by commercial ELISA tests, showing the same serological profile of animals vaccinated with a gE-deleted vaccine. Decision (2004/558/EC) does not regard bubaline animals, however this study suggests that cattle infected by this BuHV1 strain could result gB-positive by BoHV1 ELISA tests. Although herpesviruses are generally species-specific, BuHV1 infection in cattle and goats has been recently reported (Maidana et al., 2016; Camero et al., 2017) and cross-infection cannot be ruled out.

The Directive 64/432/EEC includes also the species Bubalus bubalus in the definition of bovine animals to manage animal health issues for intra-Community trade. Considering that buffaloes infected with BuHV1 can result gE-negative or gE-positive (Nogarol et al., 2014) and that interspecies transmission is possible, differentiation between animals infected by BoHV1 or by BuHV1 could be difficult by using traditional serological tests, thus the use of more specific tests should be encouraged. The risk that cattle infected with this gE-negative BuHV1 strain result seropositive for BoHV1 by ELISA tests should be better investigated because it could interfere with eradication programs approved by the European Union. The BuHV1 described in this study has been isolated from nasal and vaginal swabs from an infected buffalo after glucocorticoid treatment. The virus caused mild disease when inoculated in an uninfected and unvaccinated buffalo; it is thus possible that this virus spreads among animals without obvious clinical signs but causing seroconversion, as reported also for other different ruminant species (Maidana et al., 2016; Camero et al., 2017).

Partial sequences of the genes encoding $\mathrm{gB}$ and $\mathrm{gD}$ were obtained 


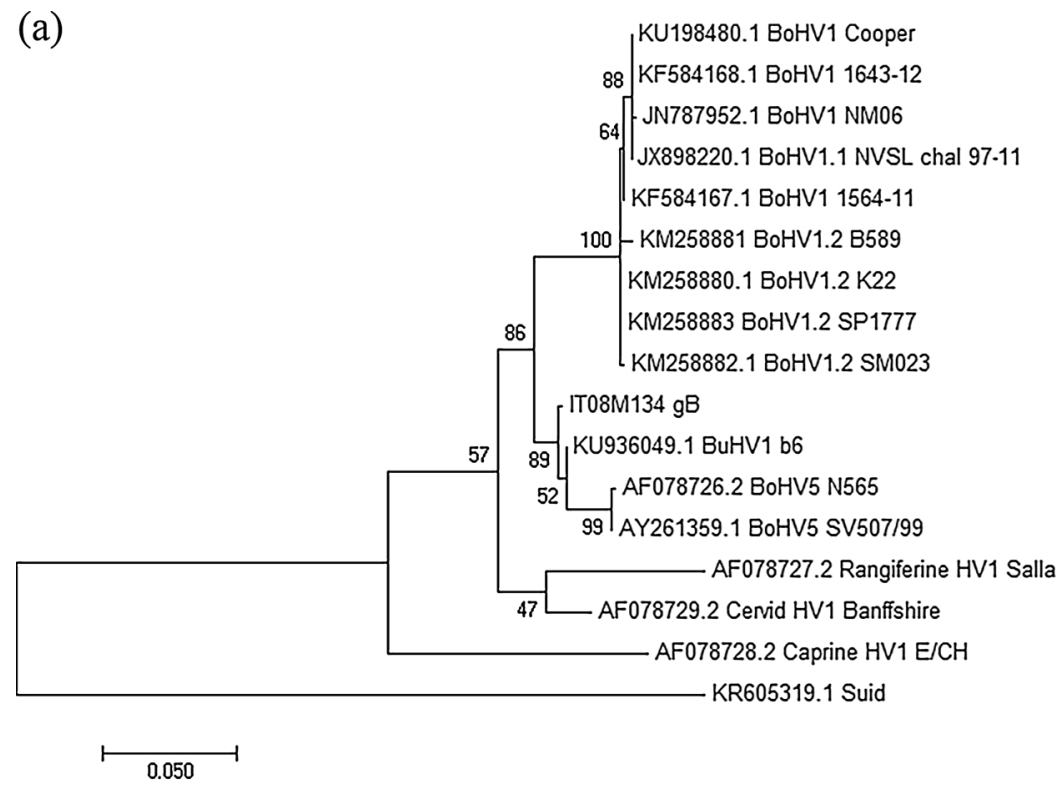

(b)

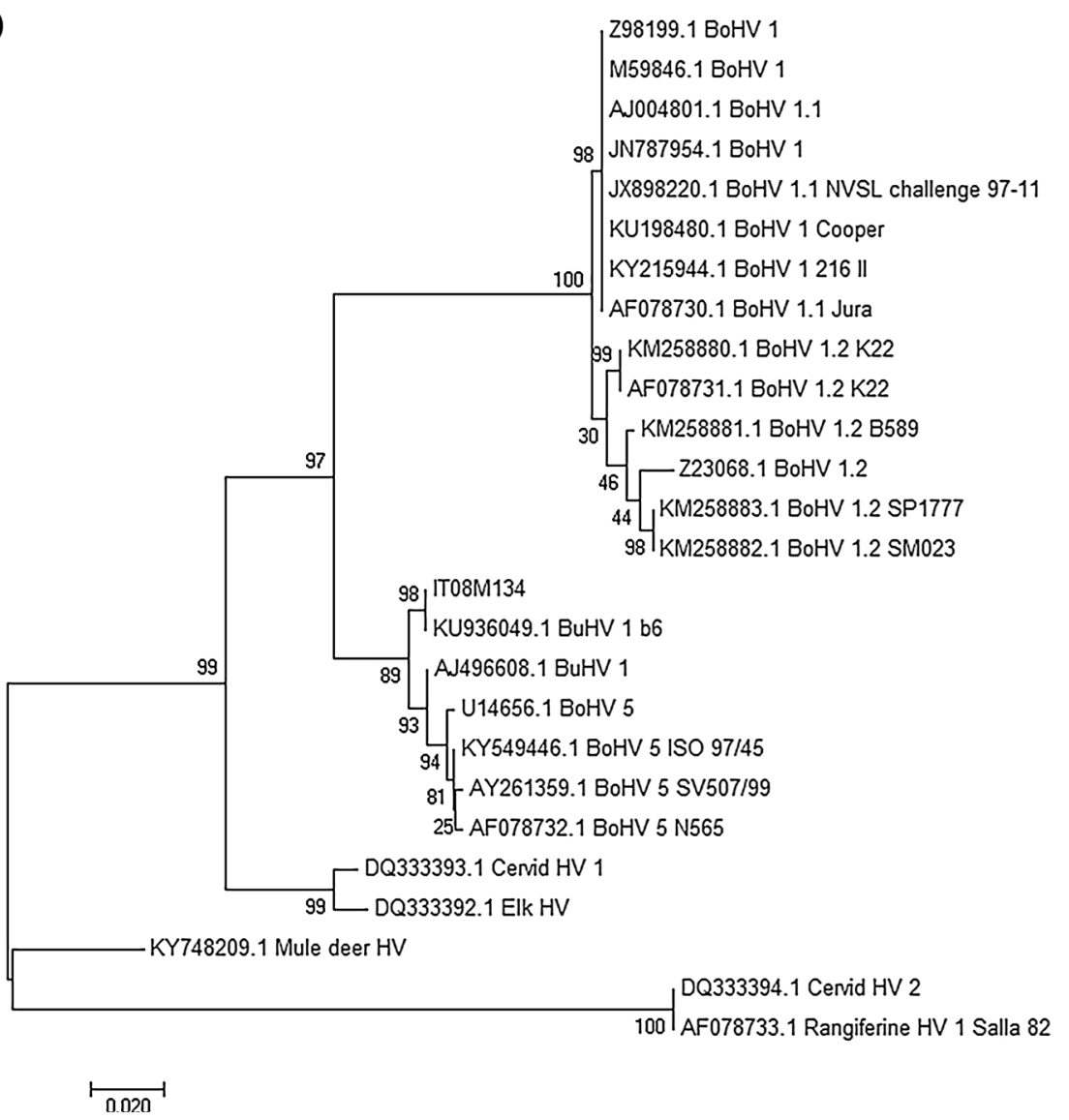

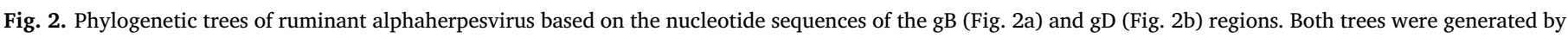

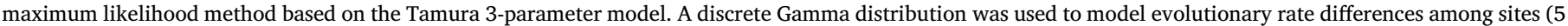
categories $(+\mathrm{G}$, parameter $=0.5486)$ ). Evolutionary analyses were conducted in MEGA7. Suid Herpesvirus 1 was used as the outgroup.

from DNA of BuHV1 strain IT08M134, while none product was obtained with primers specific for the gene encoding $\mathrm{gE}$ and described in previous studies (Fuchs et al., 1999; De Carlo et al., 2004). Phylogenetic tree based on $\mathrm{gB}$ and $\mathrm{gD}$ gene sequences clustered this strain with BuHV1, and the highest homology was found with the prototype strain b6, isolated in early 1970s in Australia from a buffalo. BuHV1 strain
IT08M134 was more closely related to BoHV-5 than to BoHV1, as previously reported for other BuHV1 strains (De Carlo et al., 2004; Thiry et al., 2007; Scheffer et al., 2017). In order to replicate previous studies, sequencing of another DNA tract was done by primers IB1 and IB3, which in bibliography are reported as specific for gI gene (Santurde et al., 1996; De Carlo et al., 2004). However, after analysis of the 
sequences obtained, IB1 and IB3 primers resulted specific for the gB gene instead of the gI gene, and the sequence obtained was mostly overlapped but shorter than the sequence obtained by $\mathrm{gB}-1 / \mathrm{gB}-2$ primers.

In conclusion, this study describes a BuHV1 strain inducing a seroconversion profile which could interfere with IBR control and eradication programs. Indeed, animals infected from BuHV1 IT08M134 result gB-positive and gE-negative when tested by commercial ELISA tests, showing a seroconversion profile similar to animals vaccinated with a BoHV1 gE-deleted vaccine. Further investigations should be aimed at sequencing the full genome of this viral isolate and at evaluating the susceptibility of other animal species.

\section{Conflict of interests}

There are no conflicts of interest in the study.

\section{Acknowledgements}

The experiments have been approved by the ethical commission of the University of Camerino (Prot. n. 4/2014).

The authors thank Lorène Dams for her excellent technical assistance in the REA tests.

\section{Appendix A. Supplementary data}

Supplementary material related to this article can be found, in the online version, at doi:https://doi.org/10.1016/j.vetmic.2018.05.013.

\section{References}

Amoroso, M.G., Corrado, F., De Carlo, E., Lucibelli, M.G., Martucciello, A., Guarino, A., Galiero, G., 2013. Bubaline herpesvirus 1 associated with abortion in a Mediterranean water buffalo. Res. Vet. Sci. 94, 813-816.

Camero, M., Larocca, V., Losurdo, M., Lorusso, E., Patruno, G., Staffa, V.N., Martella, V., Buonavoglia, C., Tempesta, M., 2017. Goats are susceptible to bubaline alphaherpesvirus 1 infection: results of an experimental study. Comp. Immunol. Microbiol. Infect. Dis. 50, 97-100.

Caruso, C., Prato, R., Ingravalle, F., Vecchio, D., Sciarra, A., Ternavasio, M., Ceccarelli, L., Martucciello, A., Galiero, G., De Carlo, E., Masoero, L., 2016. Prevalence of antibodies against bubaline herpesvirus (BuHV-1) among Mediterranean water buffalo (Bubalus bubalis) with implications in buffalo trade. Vet. Q. 36, 184-188.
Castrucci, G., Frigeri, F., Ranucci, S., Ferrari, M., Cilli, V., Pedini, B., Nettleton, P., Caleffi, F., Aldrovandi, V., Herring, A.J, 1984 Comparative studies of strains of infectious bovine rhinotracheitis virus isolated from latently infected calves. Comp. Immunol. Microbiol. Infect. Dis. 7, 1-10.

Decision 2004/558/EC of 15 July 2004 Implementing Council Directive 64/432/EEC as Regards Additional Guarantees for Intra-community Trade in Bovine Animals Relating to Infectious Bovine Rhinotracheitis and the Approval of the Eradication Programs Presented by Certain Member States.

De Carlo, E., Re, G.N., Letteriello, R., Del Vecchio, V., Giordanelli, M.P., Magnino, S., Fabbi, M., Bazzocchi, C., Bandi, C., Galiero, G., 2004. Molecular characterisation of a field strain of bubaline herpesvirus isolated from buffaloes (Bubalus bubalis) after pharmacological reactivation. Vet. Rec. 154, 171-174.

Fuchs, M., Hübert, P., Detterer, J., Rziha, H.J., 1999. Detection of bovine herpesvirus type 1 in blood from naturally infected cattle by using a sensitive PCR that discriminates between wild-type virus and virus lacking glycoprotein E. J. Clin. Microbiol. 37, 2498-2507.

Hall, T.A., 1999. BioEdit: a user-friendly biological sequence alignment editor and analysis program for windows 95/98/NT. Nucl. Acids Symp. Ser. 41, 95-98.

Kumar, S., Stecher, G., Tamura, K., 2016. MEGA7: molecular evolutionary genetics analysis version 7.0 for bigger datasets. Mol. Biol. Evol. 33, 1870-1874.

Larkin, M.A., Blackshields, G., Brown, N.P., Chenna, R., McGettigan, P.A., McWilliam, H., Valentin, F., Wallace, I.M., Wilm, A., Lopez, R., Thompson, J.D., Gibson, T.J., Higgins, D.G., 2007. Clustal W and clustal X version 2.0. Bioinformatics 23, 2947-2948.

Maidana, S.S., Delgado, F., Vagnoni, L., Mauroy, A., Thiry, E., Romera, S., 2016. Cattle are a potential reservoir of bubaline herpesvirus 1 (BuHV1). Vet. Rec. Open 3, e000162.

Nogarol, C., Bertolotti, L., De Carlo, E., Masoero, L., Caruso, C., Profiti, M., Martucciello, A., Galiero, G., Cordioli, P., Lelli, D., Nardelli, S., Ingravalle, F., Rosati, S., 2014. Expression and antigenic characterization of bubaline herpesvirus 1 (BuHV1) glycoprotein E and its potential application in the epidemiology and control of alphaherpesvirus infections in Mediterranean water buffalo. J. Virol. Methods 207, 16-21.

Ros, C., Belák, S., 1999. Studies of genetic relationships between bovine, caprine, cervine, and rangiferine alphaherpesviruses and improved molecular methods for virus detection and identification. J. Clin. Microbiol. 37, 1247-1253.

Santurde, G., Da Silva, N., Villares, R., Tabares, E., Solana, A., Bautista, J.M., Castro, J.M., 1996. Rapid and high sensitivity test for direct detection of bovine herpesvirus-1 genome in clinical samples. Vet. Microbiol. 49, 81-92.

Scheffer, C.M., Varela, A.P., Cibulski, S.P., Schmidt, C., Campos, F.S., Paim, W.P., Dos Santos, R.N., Teixeira, T.F., Loiko, M.R., Tochetto, C., Dos Santos, H.F., de Lima, D.A. Cerva, C., Mayer, F.Q., Petzhold, S.A., Franco, A.C., George, T.S., Spilki, F.R., Roehe, P.M., 2017. Genome sequence of bubaline alphaherpesvirus 1 (BuHV1) isolated in Australia in 1972. Arch. Virol. 162, 1169-1176.

Tamura, K., 1992. Estimation of the number of nucleotide substitutions when there are strong transition-transversion and G + C-content biases. Mol. Biol. Evol. 9, 678-687.

Thiry, J., Keuser, V., Muylkens, B., Meurens, F., Gogev, S., Vanderplasschen, A., Thiry, E., 2006. Ruminant alphaherpesviruses related to bovine herpesvirus 1. Vet. Res. 37, 169-190.

Thiry, J., Widén, F., Grégoire, F., Linden, A., Belák, S., Thiry, E., 2007. Isolation and characterisation of a ruminant alphaherpesvirus closely related to bovine herpesvirus 1 in a free-ranging red deer. BMC Vet. Res. 3, 26. 